\title{
Human Machine and Thermoelectric Energy Scavenging for Wearable Devices
}

\author{
Vladimir Leonov \\ Smart Systems and Energy Technology Unit, Imec, Kapeldreef 75, 3001 Leuven, Belgium \\ Correspondence should be addressed to Vladimir Leonov, leonov@imec.be
}

Received 23 August 2011; Accepted 21 September 2011

Academic Editors: S. Cook and F. E. Little

Copyright () 2011 Vladimir Leonov. This is an open access article distributed under the Creative Commons Attribution License, which permits unrestricted use, distribution, and reproduction in any medium, provided the original work is properly cited.

Thermal properties of humans were studied in the case where a small-size energy scavenger is placed on the body. In such a case, the human being serves as a heat source for the thermopile of the scavenger, but the latter serves as a thermally insulating object. As a result, the body properties, namely, the skin temperature, heat flow, and thermal resistance locally change. This is the result of redirection of heat flow inside the body to colder zones because of thermal insulation provided by the scavenger. Increased thermal resistance of human body, in turn, affects the design of the scavenger. The analysis of such scavenger performed for ambient temperatures of $0^{\circ} \mathrm{C}$ to $25^{\circ} \mathrm{C}$ shows that it could reach competitive performance characteristics and replace batteries in low-power wearable electronics. A simulated power of up to $60 \mu \mathrm{W} / \mathrm{cm}^{2}$ at $0^{\circ} \mathrm{C}$ has been validated by using wearable thermoelectric modules.

\section{Introduction}

The past years demonstrated growing interest of the world market to small hidden wearable devices for monitoring of chronic deceases and vital signs. Such devices must preferably be wireless and form a body-area network. Constant monitoring of physiological parameters will improve preventive healthcare through early detection of deceases and the parameter deviation. This will help to hold in control the gradually increasing cost of curative medicine [1]. Logically, such devices must be integrated in the things already existing on the human body such as watches, jewelry, and garments. The necessity of a battery complicates device integration and slows down wide spread of health monitoring devices on the market. However, the battery in a wearable sensor could be provided with or replaced by an energy scavenger, or harvester, that would transform available ambient energy into electrical one. Among ways of scavenging ambient energy, three could be considered: the photovoltaic conversion of ambient light, the conversion of mechanical energy of a person, which can be done through electromagnetic, electrostatic, or piezoelectric mechanisms, and the thermoelectric conversion of human heat.

Photovoltaic cells can be easily obscured from light by pieces of closing worn on top of those with cells. Therefore, their use in garments can hardly be accepted because physiological sensors are located directly on the skin, that is, they should be integrated in underwear to avoid electrical interconnections between pieces of clothing. Scavenging of mechanical energy for healthcare systems is unreliable because the patients and elderly people, that is, those who typically need healthcare, may have minimum physical activity on occasional basis. Furthermore, in mechanical systems, the produced power is directly proportional to the proof mass, that is, to the weight of the mass moving in the energy scavenger. Taking into account low frequency of human motion, the power produced by such energy-scavenging microsystem is relatively low. Consequently, scavenging of mechanical energy, which can be used for powering devices, for example, in the shoes of a sportsman is not suited for preventive healthcare. The third source of power, namely, the heat flow from humans, is suited for everyone. The human being itself generates heat that can be used to power wearable sensors. This heat generated in humans and warmblooded animals as a side effect of metabolism allows constant body core temperature and typically provides certain temperature difference between the body and environment. The resulting heat flow from human being to the ambient can be transformed into electricity through thermoelectric conversion [2]. A wearable thermoelectric energy scavenger is a reliable 
source of power. In addition, it provides unlimited service life to wireless sensors with no need for recharging or replacing batteries. Several wearable battery-less wireless devices and those with a rechargeable battery have already been demonstrated $[3,4]$. These devices are powered thermoelectrically or by using combined photovoltaic and thermoelectric energy scavenging. Their suitability for integration in clothes has also been studied $[3,5]$.

This paper discusses principles of energy scavenging with a thermoelectric converter of heat called thermoelectric generator (TEG). It is shown that the thermal properties of human being must be accounted for the TEG optimization, for reaching its maximum power and, finally, for success of self-powered devices on the market. The study of human being performed in this work shows that the wearable TEG and human body influence the properties of each other. The results obtained on thermal resistance of humans have enabled evaluation of the target performance characteristics of wearable TEGs reported below. The calculation results are being compared with experimental data.

\section{Heat Generation and Dissipation by Human Being}

Metabolism of a subject has been studied by Harris and Benedict [6]. According to their equations, the daily heat production of men and women with no physical activity and not accounting for food digestion, that is, the basal metabolism is:

$$
\begin{array}{cl}
\text { (Men) } H= & 66.470+13.7516 w \\
& +5.0033 s-6.7550 a[\mathrm{kcal}] \\
\text { (Women) } H= & 655.0955+9.5634 w \\
& +1.8496 s-4.6756 a[\mathrm{kcal}]
\end{array}
$$

where $H$ is total heat production per $24 \mathrm{hrs}, w$ is weight in kilograms, $s$ is stature in centimeters, and $a$ is age in years. Possibly, modern population, some nations, or groups of society may show certain deviation from the above equations established almost a century ago. For example, Mifflin et al. [7] arrived in the late 90s to the conclusion that, in studied Americans, the Harris-Benedict equations overestimate metabolism by $5 \%$. They also compare some other similar research, see [7] for details. Their equation for the basal metabolism is as follows:

$$
H=9.99 w+6.25 s-4.92 a+166 m-161[\mathrm{kcal}]
$$

where $m$ is sex-related coefficient, 1 for men and 0 for women. A deviation in metabolic rate of up to $30 \%$ from any of the above equations is possible for some subjects. This unpredictable personal deviation could be due to individual differences in genetically determined or acquired metabolic efficiency. The above equations are valid for adult population.

The basal metabolic rate results in a heat flow inside the body corresponding to about $58.2 \mathrm{~W}$ per square meter of the body area. This heat flow is the commonly accepted measure of resting energy expenditure for an individual and it corresponds to the metabolic equivalent (MET). The latter is defined as $1 \mathrm{kcal} \mathrm{kg}^{-1} \mathrm{hr}^{-1}$, or as an oxygen uptake at a rate of $3.5 \mathrm{~mL} \mathrm{~kg}^{-1} \mathrm{~min}^{-1}$. Actual heat flow on the skin of resting subject is less than $58.2 \mathrm{~W} \mathrm{~m}^{-2}$, because some heat is lost through inhalation, and some heat is rejected into the ambient from the skin in a form of latent heat of vaporization.

The metabolic heat of a subject depends on his physical activity. Like in any other machine, moving results in heat generation. However, the efficiency of human machine is well above Carnot engine. At typical temperature difference between body core and the ambient of $15^{\circ} \mathrm{C}$, that is, at Carnot efficiency of $0.5 \%$, the human machine shows about 40 times better performance. As an example, we can compare results of stationary bicycling. At very light effort $(50 \mathrm{~W}$ mechanical load), the subject metabolic rate is $3 \mathrm{MET}$, while at very vigorous effort, $250 \mathrm{~W}$, the metabolic rate increases to $12.5 \mathrm{MET}$. The difference of 9.5 MET results in increase of mechanical output by $200 \mathrm{~W}$, which corresponds to $20 \%$ efficiency of a subject with body area of $1.8 \mathrm{~m}^{2}$. There appears $800 \mathrm{~W}$ of additional heat to be dissipated. However, in typical situations, most of this excess heat is rejected from the body through sweating, that is, the heat flow on the person's skin only slightly increases, even at vigorous effort (frequently, it decreases because of sweating). Furthermore, some increase of heat flow from the skin during exercise is mainly attributed to movement of subject in respect to air, that is, due to forced air convection. While extremities show vasomotor response and typically change skin temperature during exercise, the skin temperature in trunk stays practically the same. Therefore, physical exercise does not improve heat flow from the trunk, but evaporation.

In case of wearable thermoelectric generator, the heat flow from the skin to the ambient is typically the only relevant heat rejection mechanism. There are two main ways of such heat dissipation with no sweating, namely, radiation heat transfer and convection. The former takes place in long-wave infrared (LWIR) spectral region, where emission coefficient of human skin is $97-98 \%$ irrespective of race. Although in direct sunlight the skin gains more energy than that reemitted in LWIR, most of the time people do not spend in direct sunlight, but indoors, where the power emitted in LWIR from open skin, according to the Planck's law, is as follows:

$$
W_{\text {rad }}=\varepsilon \sigma\left(T_{\text {skin }}^{4}-T_{\mathrm{amb}}^{4}\right) A,
$$

where $\varepsilon$ is emission coefficient, $\sigma$ is the Stefan-Boltzmann constant, $T_{\text {skin }}$ and $T_{\mathrm{amb}}$ are temperatures of the skin and ambient in Kelvin, and $A$ is the effective radiating area. A nude person sitting still indoors looses about a half of heat flow from skin in a form of radiation, and the other half through convection and sweating. Clothes form some barrier for LWIR. They absorb body's radiation and reemit it into the ambient, but at lower temperature than that of skin. As far as temperature in (3) is to the power of four, clothes decrease radiation heat exchange. A similar effect clothes have on 
convection, either natural or forced. Natural convection is observed on a still person in quiescent air. The air heated by the body moves upwards being driven by buoyancy force. In the wind, or on a moving person, a forced convection is observed instead. Convection from such objects like human body, which has complex shape, nonuniform temperature, and in addition is partially covered by thermally insulating clothes, cannot be described by a simple equation. There are, however, some empirical correlations that enable evaluation of convective heat transfer from humans [8].

In this paper, a relatively small object, namely, a thermopile is supposed to be on the body. The electrical power, $P$, transferred by a thermopile into electrically matched load depends on the heat flow, $W$, and thermoelectric efficiency of its transformation into electricity, $\eta$ :

$$
\begin{gathered}
P=W_{\eta}, \\
\eta=\frac{Z \Delta T_{\mathrm{tp}}}{4},
\end{gathered}
$$

where $Z$ is the thermoelectric figure-of-merit, and $\Delta T_{\mathrm{tp}}$ is the temperature drop on the thermopile that must be a thermally insulating component. Indeed, ambient air shows high thermal resistance, $R_{\mathrm{amb}}$. High thermal resistance of human body, $R_{h}$, is observed in medical studies; see, for example, [8-10]. If the thermopile has a thermal resistance, $R_{\mathrm{tp}}$, much less than $\left(R_{\mathrm{amb}}+R_{h}\right)$, the temperature drop on it, $\Delta T_{\mathrm{tp}}$, will be small. As a result, $\eta$, according to (5), will approach zero, and the power, according to (4), will be low. However, if a thermopile has $R_{\mathrm{tp}} \gg R_{\mathrm{amb}}+R_{h}$, the efficiency maximizes, but heat flow minimizes. The product of a good efficiency by near-zero heat flow will be low again, according to (4). Therefore, the power maximizes in case where the thermopile has a thermal resistance of the order of those of the body and air. This unavoidably changes the heat flow from the skin under the thermopile. Consequently, the thermal resistance of body under such thermopile changes locally and the data measured in medical literature, for example, in [8-10], are not applicable to the case of a human being used as a heat source for power generation. Before taking a closer look at the interaction of the human body with a TEG, let us define the conditions that enable reaching maximum power.

\section{Thermoelectric Conversion in a Wearable Energy Scavenger}

A TEG functions, in the thermal domain, as a load of thermal generator. In case of human being as a heat generator, the thermal generator is composed from thermal resistances of the body and ambient air, Figure 1, that is, of the heat source and sink. They are connected in series to each other:

$$
R_{\text {th.gen }}=R_{\mathrm{amb}}+R_{h} .
$$

The thermal resistance of interfaces and heat conducting components of the TEG must also be included into $R_{\text {th.gen }}$. These can be made much lower than $R_{\mathrm{amb}}$ and $R_{h}$, therefore, they are omitted in Figure 1. A thermopile is a fragile object and, typically, a TEG has some other holding or encapsulation elements, which are responsible for some parasitic heat flow between hot and cold sides of a TEG. Therefore, the thermal resistance of a TEG, $R_{\mathrm{TEG}}$, can be, in the simplest case, represented by two parallel thermal resistors:

$$
R_{\mathrm{TEG}}=\frac{1}{\left(1 / R_{\mathrm{tp}}+1 / R_{\mathrm{par}}\right)},
$$

where $R_{\text {par }}$ accounts for both the heat conduction through the air inside the TEG and radiation heat exchange between its hot and cold components. From (4) and (5), the maximum power is generated when the product $W \Delta T_{\mathrm{tp}}$ maximizes. In the following discussion, the skin temperature, $T_{\text {skin }}$, is assumed to be equal to the temperature of hot thermopile junctions, while the outer heat exchanging plate of the TEG has the same temperature, $T_{\text {ext }}$, as cold junctions, so that $\Delta T_{\text {tp }}=T_{\text {skin }}-T_{\text {ext }}$. Then, the maximum power, $P_{\max }$, generated by an optimized TEG can be expressed as follows:

$$
P_{\max }=\frac{Z \Delta T_{\mathrm{tp}, \mathrm{opt}}^{2}}{16 R_{\text {th.gen }}\left(1+N^{-1}\right)},
$$

where $N=R_{\text {par }} / R_{\text {th.gen }}$ is the thermal insulation factor. It shows how well the TEG is suited for the application in the particular thermal environment; the greater $N$ is, the higher the power. However, at $N$ of one, only a half of maximum power can be obtained.

The heat flow, $W$, in the thermal circuit shown in Figure 1 depends on the thermal resistance of a TEG:

$$
W=\frac{\Delta T}{\left(R_{\text {th.gen }}+R_{\mathrm{TEG}}\right)},
$$

where $\Delta T=T_{\text {core }}-T_{\mathrm{amb}}, T_{\text {core }}$ is deep body temperature or core temperature. The latter stays typically around $37^{\circ} \mathrm{C}$ in a healthy subject. According to (9), practically any TEG placed on the body changes heat flow. The optimal temperature drop on a thermopile at maximum power equals to

$$
\Delta T_{\text {tp }, \mathrm{opt}}=\frac{\Delta T}{\left[2\left(1+R_{\text {th.gen }} / R_{\mathrm{th}, \mathrm{em}}\right)\right]},
$$

where $R_{\text {th,em }}$ is the thermal resistance of "empty" TEG, which would be observed if the thermal conductivity of thermoelectric materials was equal to that of air. It is approximately equal to the parasitic thermal resistance observed in a TEG, $R_{\text {par }}$, connected in parallel to the thermopile, $R_{\text {th,em }} \approx$ $R_{\text {par. }}$. It is related to the thermal conductance of the air inside a TEG, of thermally insulating encapsulation, and of holding components located between the plates of TEG. The optimal temperature drop does not depend on properties of thermoelectric materials. However, it depends on parasitic thermal resistance, and on the $R_{\text {th.gen }} / R_{\text {par }}$ ratio. Therefore, the thermal design of the TEG and its interfaces with the human body and heat sink are of primary importance. While replacing $R_{\text {th,em }}$ with $R_{\text {par }}$, the following equation of thermal matching was obtained in [11] that enables reaching the power maximum in a wearable TEG:

$$
R_{\mathrm{TEG}, \mathrm{opt}}=\frac{R_{\mathrm{th.gen}} R_{\mathrm{par}}}{\left(2 R_{\mathrm{th.gen}}+R_{\mathrm{par}}\right)},
$$



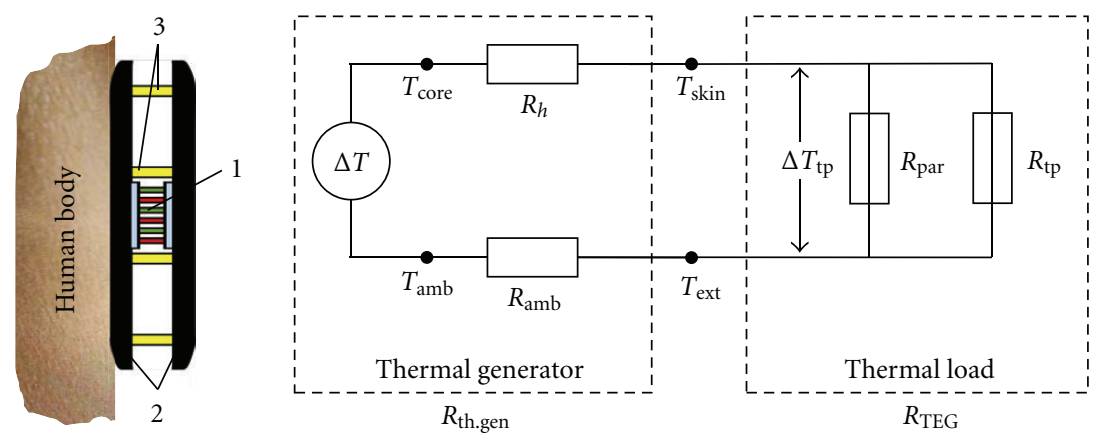

Figure 1: An example of a TEG on the human body and its thermal circuit: (1) is a thermopile between (2) two plates supported by (3) holding and encapsulating elements.

where $R_{\mathrm{TEG}, \mathrm{opt}}$ is the optimal thermal resistance of a TEG required for reaching maximum power. The thermal resistance of the thermopile at maximum power, $R_{\mathrm{tp}, \mathrm{opt}}$, can be obtained from two thermal resistors in a TEG connected in parallel to each other:

$$
R_{\mathrm{tp}, \mathrm{opt}}=\frac{R_{\mathrm{par}} R_{\mathrm{TEG}, \mathrm{opt}}}{\left(R_{\mathrm{par}}-R_{\mathrm{TEG}, \mathrm{opt}}\right)} .
$$

Note that Peltier effect increases thermal conductivity of a thermopile. Therefore, $R_{\text {tp,opt }}$ is the effective thermal resistance that accounts for electrical contribution, namely, the Peltier effect and Joule heat. The latter is, however, not significant in a wearable device and has been neglected in the simulations performed in this work.

Among the parameters required for thermal optimization of a TEG, the thermal resistance of human being is the least studied one. Its measurement was one of the main tasks of this work, and the results are reported below. Despite the fact that the thermal properties of humans have extensively been studied in medicine [8-10], the obtained results cannot directly be used for designing wearable TEGs. This is because the thermal resistance of a TEG, according to (11), must be high in the optimized device, while in such case heat flow depends on its thermal resistance, see (9). The above equations are suited for any thermopile application including those on heated machinery. However, the discussion in this paper is limited by wearable devices.

\section{The Human Machine as the Heat Supply for a TEG}

Unlike machinery, the human body has relatively high thermal resistance. Typically, the lower the ambient temperature, the higher the thermal resistance of the body. Therefore, the heat flow is quite limited. This is because warmblooded animals and man have received in the process of evolution a very effective thermal management. In particular, a very high thermal resistance of the body is observed at ambient temperatures below $15-20^{\circ} \mathrm{C}$ in case where skin temperature decreases below the sensation of thermal comfort. This does not happen if appropriate clothing is worn to maintain the heat balance near thermal comfort. At typical indoor conditions, the location-dependent heat flow from a person mainly stays within the 1 to $10 \mathrm{~mW} / \mathrm{cm}^{2}$. The open skin surface (the face and, above $20-25^{\circ} \mathrm{C}$, hands) produces larger heat flow per unit area than the body parts thermally insulated by clothes. Due to garments, heat dissipation from the areas covered by textile is typically below 4$5 \mathrm{~mW} / \mathrm{cm}^{2}$. Depending on physical activity, clothes, and ambient conditions (temperature, wind, and humidity), the heat dissipation in extremities either enhanced or minimized. This is one of the important mechanisms of body temperature regulation for preservation of the body core temperature at low metabolic rate or in cold environment, and for dissipation of excess heat if the body temperature dangerously rises due to vigorous physical effort or on a hot day.

The TEGs were used in this work as a useful tool for measuring location-dependent thermal properties of the human being, that is, of the heat flow and local thermal resistance, $R_{h}$. The generated open-circuit voltage allows determination of the temperature drop on a thermopile. The latter, being divided by the thermal resistance of a thermopile, gives the heat flow. The skin temperature under the TEG changes if the heat flow through the device differs from the heat flow observed around the device. This is a typical situation, because according to the theory discussed above, the thermal resistance of the optimized TEG must be high, of the order of hundreds $\mathrm{cm}^{2} \mathrm{~K} / \mathrm{W}$. Therefore, heat flow measurements have been accompanied by the measurement of the hot plate temperature. Therefore, the measured $R_{h}$ includes the interface thermal resistance between the skin and TEG. Five TEGs used in this work are shown in Figure 2. Relevant specifications are listed in Table 1.

The dependence of the thermal resistance of human body on heat flow has been studied in this work in two locations: on the radial artery of the left wrist and on the anterior side of the left leg, about $25 \mathrm{~cm}$ above the knee. The experiments have been conducted indoors, in the office, at temperatures within $21.0^{\circ} \mathrm{C}$ to $23.7^{\circ} \mathrm{C}$, with no air conditioning, on a sitting person, in a course of several days. The subject was wearing a shirt and jeans. The TEG-4 with hot plate area of $7 \mathrm{~cm}^{2}$ was attached to the body by using elastic band. For the measurements on the leg, a hole was cut in the jeans. The outer plate of the TEG was cooled to different extent, 

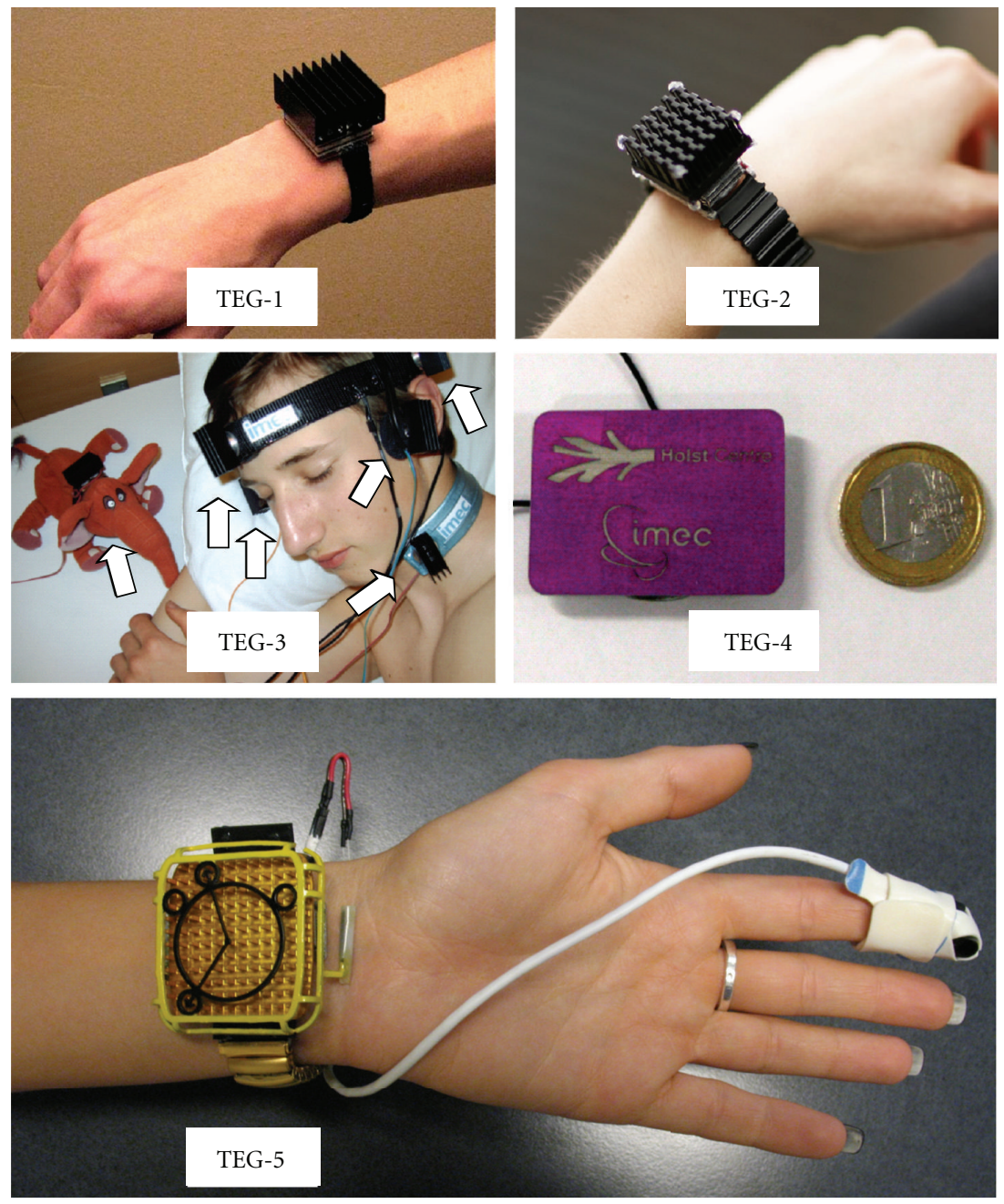

FIGURE 2: Thermoelectric generators used for the measurement of thermal properties of human body on the trunk, head, and extremities. The shown TEG-5 powers $\mathrm{SpO}_{2}$ wireless sensor [12].

and the open-circuit Seebeck voltage was measured. It is proportional to heat flow. Therefore, the heat flow was obtained from the Seebeck voltage and thermal resistance of the thermopile, $R_{\mathrm{tp}}$, although accounting for parasitic heat flow through the holding and encapsulating elements, and the air inside the TEG. A thermocouple glued to the plate of TEG touching the skin was used for monitoring skin temperature. The core temperature has been evaluated based on both deep oral and tympanic temperatures measured periodically by the infrared thermometer with a resolution of $0.1^{\circ} \mathrm{C}$. Slow drifting of the temperature of a large part of an extremity during one hour, and longer was observed in the experiment in case of high heat flows, that is, those essentially exceeding the natural heat flow. Therefore, to minimize this uncertainty, another experiment has been conducted with halved contact area of the TEG with the body, namely, on $1.6 \times 2.1 \mathrm{~cm}^{2}$ area. At the same heat flow per unit area, the heat flow through the device was halved, and the temperature stabilization was obtained much faster. The results are shown in Figure 3.
Before discussion of the data shown in Figure 3, it must be understood that the thermal resistance of any selected part of the body, or fixed volume of the body, would not change so drastically on heat flow. Mainly, only variations in blood flow would cause variation in the thermal resistance of the body. Actually, the thermal resistance shown in Figure 3 is not a net body property. It is the thermal resistance of the body associated with the heat transfer from the body into the TEG. The latter is a small-size object, so if the heat flow through the TEG decreases below the heat flow observed on the body surface not covered with a TEG, the heat from the body part covered by the TEG partially flows to colder zones of the skin. This effectively changes the geometry of the body part under the TEG responsible for the heat transfer to the TEG, and results in higher thermal resistance seen on the left side of Figure 3. In contrary, if the heat flow through the TEG exceeds the heat flow through the neighboring skin area next to the TEG, the latter effectively collects heat from the body part exceeding the footprint of TEG. Therefore, Figure 3 shows the thermal resistance of the body relevant only to 
TABLE 1: Relevant dimensions of TEGs shown in Figure 2 and the thermal resistance of thermopiles.

\begin{tabular}{|c|c|c|c|c|c|}
\hline \multirow{2}{*}{ Parameter } & \multicolumn{5}{|c|}{ Thermoelectric generators } \\
\hline & TEG-1 & TEG-2 & TEG-3 & TEG-4 & TEG-5 \\
\hline $\begin{array}{l}\text { Dimensions of the hot } \\
\text { plate, } \mathrm{cm}\end{array}$ & $3 \times 3$ & $2.1 \times 2.4$ & $4 \mathrm{~cm}^{2}$ (circular) & $7 \mathrm{~cm}^{2}$ (circular) & $6 \mathrm{~cm}^{2}$ \\
\hline $\begin{array}{l}\text { Dimensions of the } \\
\text { radiator, } \mathrm{cm}\end{array}$ & $1.6 \times 3.6 \times 3.8$ & $1.55 \times 2.9 \times 3$ & $1.6 \times 1.6 \times 3.8$ & $3 \times 4$ (plate $)$ & $11.5 \mathrm{~cm}^{2} \times 0.5$ \\
\hline $\begin{array}{l}\text { Thermal resistance of } \\
\text { thermopiles, K/W }\end{array}$ & 16.5 & 25 & 38 & 76 & 35 \\
\hline
\end{tabular}

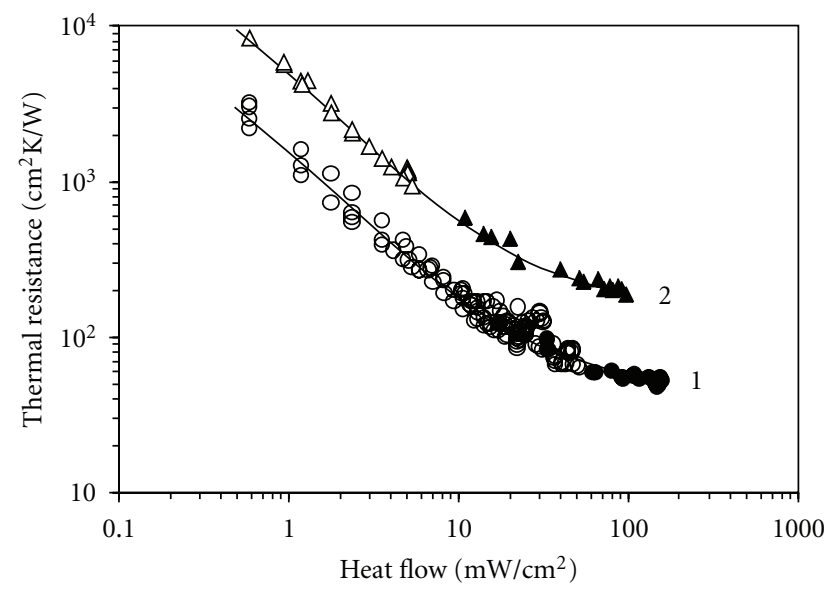

Figure 3: Dependence of the thermal resistance of the (1) wrist and (2) leg on heat flow measured in the office. The results were obtained on the area of $7 \mathrm{~cm}^{2}$ (open points) and $3.4 \mathrm{~cm}^{2}$ (closed points).

the heat flow transfer from the body into the TEG, and thus required for modeling of a wearable energy scavenger. It can be used neither for description of the human being itself nor for natural heat transfer from human being with no attached TEG.

The results shown in Figure 3 for two studied locations reflect two different situations on the human body. On the leg, the thick layer of muscles between an artery and TEG resulted in a much higher thermal resistance than on the wrist, where the TEG was located at a few millimeters from the radial artery. The muscles are the major thermal insulator of human body. In contrary, arteries are effective heat spreaders and excellent heat exchangers. Therefore, much higher heat flow was reached through the TEG on the wrist. At high heat flow, the thermal resistance in both locations seems to stabilize. It reaches minima of about $200 \mathrm{~cm}^{2} \mathrm{~K} / \mathrm{W}$ on the leg and $60 \mathrm{~cm}^{2} \mathrm{~K} / \mathrm{W}$ on the wrist. Typically, the heat flow observed on human skin indoors is much less than $10 \mathrm{~mW} / \mathrm{cm}^{2}$. The average heat flow can be estimated from the metabolic activity and the subject body area. For example, a person working in the office at a metabolic rate of 1.2 MET, produces $7 \mathrm{~mW} / \mathrm{cm}^{2}$, but about $2 \mathrm{~mW} / \mathrm{cm}^{2}$ are rejected in a form of latent heat of sweat vaporization. Therefore, the heat flow on skin is about $5 \mathrm{~mW} / \mathrm{cm}^{2}$, on average. It is higher on open skin area, for example, on the face and neck, but it decreases on the trunk and the parts of extremities covered by cloth. At such low heat flow, the thermal resistance of the body, according to Figure 3, is reversely proportional to heat flow, may reach high values and can even exceed the thermal resistance due to ambient air. Therefore, a wearable TEG must be optimized accounting for the thermal resistance of human body corresponding to the observed heat flow. From Figure 3, at a heat flow of less than $10 \mathrm{~mW} / \mathrm{cm}^{2}$, the thermal resistance in the measured locations can be approximated as $R_{h}=1.6 / W\left(\mathrm{~cm}^{2} \mathrm{~K} / \mathrm{W}\right)$ for the TEG on the wrist, and as $R_{h}=5.1 / \mathrm{W}$ on the leg. As far as curve 1 corresponds to the device in proximity to an artery, and curve 2 is related to the device in the location with the thickest layer of muscles, far from arteries, one may believe that, under typical conditions in the office, the thermal resistance of any part of a subject's body should mainly stay between these values. However, this conclusion is supposed to be valid only in case of a person at thermal comfort. In case of vasoconstriction, distal extremities will obviously show much higher thermal resistance than those shown in Figure 3.

\section{The Human Being at Different Ambient Temperatures}

As has been found in previous section, the thermal resistance of human being depends on heat flow. The heat flow changes with the ambient temperature and air speed, for example, because of wind, or because the person walks. At fixed ambient conditions, the radiator of wearable TEG enhances heat flow, the larger the radiator, the higher the heat flow. The thermal resistance of the ambient air and body also vary depending on the radiator size. Therefore, to measure heat flows from a person and his/her thermal resistances in different locations on the body, at different ambient conditions, and on radiator size, the five different TEGs shown in Figure 2 have been used in the experiment.

The first experiment was conducted on sedentary people in the office. The heat flow and thermal resistance of the wrist was measured by wearing the TEG-1 as shown in Figure 2. All groups of participants performed exactly the same physical activities indoors (mainly, sedentary) whole day, and arrived to the laboratory at $4 \mathrm{p} . \mathrm{m}$. to $6 \mathrm{p} . \mathrm{m}$. The heat flow enhanced by the radiator was $19 \mathrm{~mW} / \mathrm{cm}^{2}$, on average. However, it depended on skin temperature. The latter was $30 \pm 2.5^{\circ} \mathrm{C}$. There was some correlation of mean heat flow and the skin temperature. The former showed variations from 15 to 
$24 \mathrm{~mW} / \mathrm{cm}^{2}$. The observed difference between subjects was relatively large, with a standard deviation of $17 \%$. Therefore, the body thermal resistance largely varied from person to person. In $90 \%$ of studied subjects, the thermal resistance of the body was within the $200-650 \mathrm{~cm}^{2} \mathrm{~K} / \mathrm{W}$ range. Its average value measured at $22.7^{\circ} \mathrm{C}$ on 77 subjects sitting still for a few tens of minutes was $440 \mathrm{~cm}^{2} \mathrm{~K} / \mathrm{W}$. The statistical study has shown that there were no correlation between the skin temperature and properties of the clothing ensembles worn by participants. It was not related to the personal sensation of comfort (cold/mild, cold/neutral/slightly, and warm/warm) either. Therefore, the difference in thermal resistance was actually related with the difference in metabolic and sweating rates, and in their build. Monitoring of heat flow on three subjects during 24 hours has shown that heat flow was $23.2 \mathrm{~mW} / \mathrm{cm}^{2}$, on average, which was larger by $23 \%$ than sedentary people.

An artery is the best heat exchanger for a wearable TEG. Therefore, another set of measurements has been performed on the people wearing the same TEG-1 on the radial artery, that is, on the inner side of the wrist. The registered heat flow was $24.8 \mathrm{~mW} / \mathrm{cm}^{2}$, on average, which was higher by $32 \%$ than on the outer side of the wrist [13]. However, low thermal resistance of thermopiles in the TEG-1 and large heat flow frequently caused complaints (in 55\% of studied cases) that the device was not comfortable even indoors. Therefore, the thermal resistance of the other TEGs used in this work has been increased to successfully avoid sensation of cold. Higher thermal resistance of a TEG allows larger temperature drop on thermopiles. Thereby it provides better thermal insulation of skin and more frequently maintains skin temperature at comfortable level even in chilly weather.

The third set of experiments with the TEG-1 worn on the radial artery has been conducted in the wide range of ambient temperatures. The measured heat flow through the TEG is shown in Figure 4 for three cases: $(1,2)$ correspond to a sedentary person in the office, but at different metabolic rates, and (3) to a walking person.

As shown in Figure 4, the heat flow through the TEG changes its direction at ambient temperature of about $36^{\circ} \mathrm{C}$. At higher ambient temperatures, the heat is absorbed from the ambient and transferred through the TEG to the arterial blood. Then, it is spread to the hand and rejected from the body back to the ambient through sweating. The difference between cases (1) and (2) at lower temperatures is explained by vasoconstriction at insufficient metabolic rate, so-called effect of cold hands. The increase of heat flow from a walking person, the curve (3), is not related to the increase in metabolic rate, but to forced air convection. The walks were relatively short (several minutes) and did not influence the body heat content. Further increase of airspeed would increase the heat flow only if the proper clothes are worn. For example, bicycling in a light clothing ensemble at ambient temperature of $16^{\circ} \mathrm{C}$ has caused $20 \%$ decrease of heat flow after $3 \mathrm{~min}$ of exercise, which is explained by cooling of the whole body by airflow and, as a result, by decreased temperature of arterial and venous blood in hands.

In the fourth set of experiments, the TEG-1, TEG-2, and TEG- 4 have been used for the measurement of the

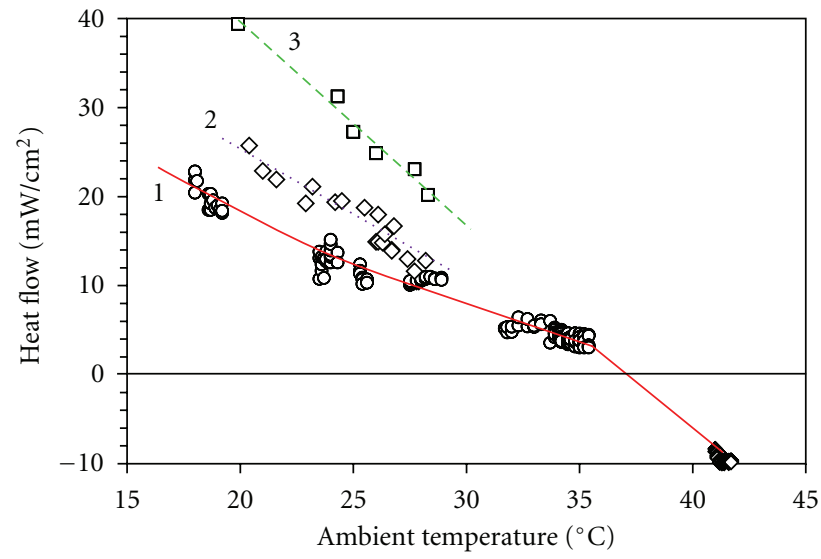

Figure 4: Dependence of the heat flow from the wrist on ambient temperature measured by using the TEG- 5 worn on the radial artery: (1) on a subject sitting for hours in the office at a metabolic rate of $1 \mathrm{MET}$, (2) on the same subject performing typical activity in the office at a metabolic rate of 1.2 MET, and (3) on the subject walking indoors, that is, at forced air convection. The lines are the guides for eyes.

body's thermal resistance within the extended range of ambient temperatures. Appropriate clothing ensemble has been chosen by the studied subject and changed, on necessity, to feel comfortable at different ambient conditions. Gloves were worn at temperatures below $5^{\circ} \mathrm{C}$. As shown in Figure 5, at low ambient temperatures, the body thermal resistance only slightly increases on the wrist, but essentially decreases on the leg. The TEG-2 is relatively bulky and therefore seems unrealistic as a wearable device. However, the radiator with the same thermal resistance can be made much thinner, for example, using large-area piece of carbon fabric integrated in garments [5]. The heat flow in TEG-4 at equal ambient conditions was lower than in TEG-2. For example, the TEG4 showed four-times lower heat flow on the leg. The thermal resistance of human body, in contrary, increases at decreased heat flow. On the wrist, it increased under the TEG-4 by about $50 \%$, and on the leg by a factor of two as compared with TEG-2.

Among other locations for a TEG, the trunk was recently studied as a heat source. It was found that it shows very broad range for thermal resistances, and right particular location must be chosen while designing the wearable TEG integrated in textile on the trunk. The measurements using TEG-4 have shown fourfold variation of the thermal resistance over the anterior trunk [1]. In Table 2, the lowest thermal resistance measured on the chest is reported together with the data obtained in other locations.

When an appropriate clothing ensemble is chosen, wearable TEGs supplied with a radiator enable large heat flow, especially at low ambient temperatures and forced convection (wind, walking). As measured at ambient temperatures of $-4^{\circ} \mathrm{C}$ to $+3^{\circ} \mathrm{C}$, they do not cause sensation of cold at a heat flow of 25 to $130 \mathrm{~mW} / \mathrm{cm}^{2}$, depending on location chosen on the body. In the experiment at ambient temperature of $-2^{\circ} \mathrm{C}$, the TEG-2 worn on the wrist unobtrusively reached a record power of $1.2 \pm 0.2 \mathrm{~mW} / \mathrm{cm}^{2}$. Indoors, the power 


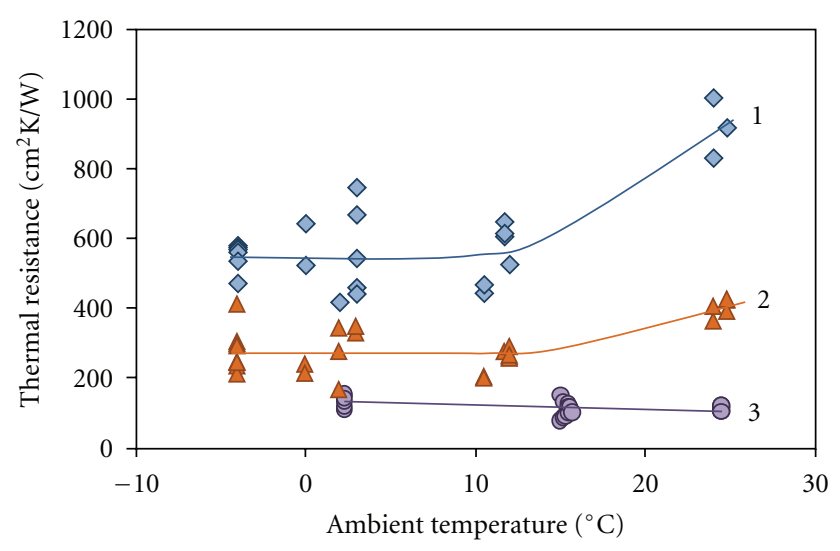

Figure 5: Dependence of the thermal resistance of the leg $(1,2)$ measured with TEG-4 (1) and TEG-2 (2), and of the wrist (3) measured with TEG-2 on ambient temperature. The results have been obtained on a standing or sitting subject. The lines are the guides for eyes.

TABLE 2: Thermal resistance in different locations measured with different TEGs.

\begin{tabular}{lccc}
\hline \multirow{2}{*}{ Location } & \multicolumn{2}{c}{$\begin{array}{c}\text { Thermal resistance, } \mathrm{cm}^{2} \mathrm{~K} / \mathrm{W} \\
\text { Indoors }\end{array}$} & $\begin{array}{c}\text { The TEG used for } \\
\text { measurements }\end{array}$ \\
\hline Wrist (dorsal) & 440 (avg.) & - & TEG-1 \\
Wrist (radial & 110 & 140 & TEG-1 \\
artery) & 160 & - & TEG-4 \\
Chest & 130 (min.) & 140 (min.) & TEG-2 \\
& 200 (min.) & - & TEG-4 \\
Forehead & $160-230$ & - & TEG-3 \\
& 380 & - & TEG-4 \\
Leg (anterior) & 400 & 280 & TEG-2 \\
& 900 & 550 & TEG-4 \\
\hline
\end{tabular}

produced by a watch-thick device like the TEG-5 typically does not exceed 25 to $30 \mu \mathrm{W} / \mathrm{cm}^{2}$ unless the person walks. Figure 6 gives an idea about possible level of conversion efficiency in the optimized wearable TEG supplied with a radiator, at different ambient temperatures. The shown data have been obtained using the TEG-2 worn on the wrist and using the TEG of an electroencephalography system with a hybrid thermoelectric-photovoltaic power supply worn on the head like a diadem [14].

\section{Expected Characteristics of Wearable Thermoelectric Generators}

In this section, the design and shape of wearable TEG is discussed, and its top performance characteristics are simulated. To be unobtrusive, the device must be relatively thin and small. A thin or small device produces less power, but can unobtrusively be integrated in garments. As discussed in previous section, the radiator attached to the thermopile decreases thermal resistance of ambient air and increases heat flow. Therefore, logically, the fins or pins of radiator improve power per unit area of TEG occupied on skin. Such devices

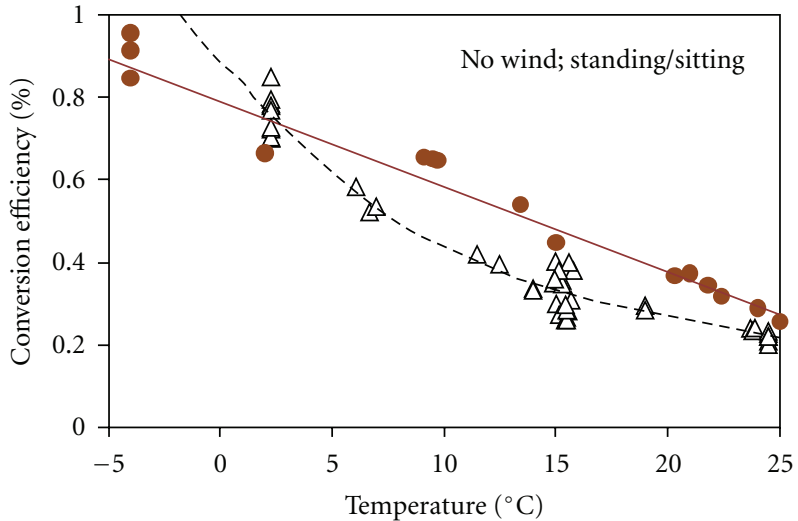

FIgure 6: Dependence of the conversion efficiency of a TEG with a radiator on ambient temperature measured on the wrist (triangles) and head (circles) of a standing or sitting person.

resembling a watch, indeed, produce usable power of 20 $30 \mu \mathrm{W} / \mathrm{cm}^{2}$ indoors [1]. But it is impossible to integrate such a thick device in a piece of clothing unobtrusively.

In the first simulation, it has been assumed that the device resembles a coin and has no pins or fins on the cold plate. By using a moderate value of $250 \mathrm{~cm}^{2} \mathrm{~K} / \mathrm{W}$ for the thermal resistance of human being, the dependence of performance characteristics of a TEG module on its thickness has been calculated. The simulation was conducted for a TEG of $3 \mathrm{~cm}$ in diameter shown in Figure 7(a). It also very much resembles the TEG-4, except the shape of its cold plate. The modeled device resembles a big coin, or a stack of such coins. An off-the-shelf thermopile is mounted between two $1 \mathrm{~mm}$-thick Al plates of $3 \mathrm{~cm}$ in diameter. Therefore, the minimum thickness of the TEG is $2.5 \mathrm{~mm}$. A state-ofthe-art $Z$ of $0.003 \mathrm{~K}^{-1}$ was assumed, which is reached in thermopile production. Because of low temperature drop on a wearable thermopile, the Joule heating was neglected. In the calculations, the thickness of the module was varied within the 2.5 to $10 \mathrm{~mm}$. The optimization was performed for every particular combination of the module thickness and ambient temperature to obtain maximum power. The modeling was conducted in case of quiescent air, that is, on a standing or sitting person. The output voltage is not critical and will not be discussed because there are effective ways of upconversion of, for example, $20 \mathrm{mV}$ produced by an offthe-shelf thermopile to the voltage required for a particular application [15]. Therefore, the quantity of thermocouples in a TEG is not important. The important parameters are the thermal resistances of the TEG and thermopile, which must correspond to (11) and (12), respectively. In the simulation, the heat transfer coefficient on the device is assumed to be equal to that on the clothed human being. The subject wearing the TEG has a core temperature of $37^{\circ} \mathrm{C}$. It is also assumed that the thermal resistance of supports and encapsulation is $400 \mathrm{~K} / \mathrm{W}$ per $1 \mathrm{~mm}$ distance between the plates, and the emission coefficient of the outer surface of the radiator is $90 \%$. The radiation inside the TEG is neglected because polished $\mathrm{Al}$ plates have very low emission coefficient. 


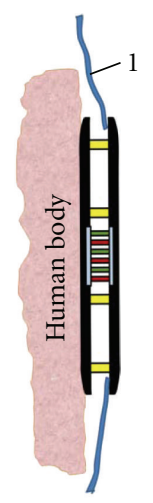

(a)



(b)

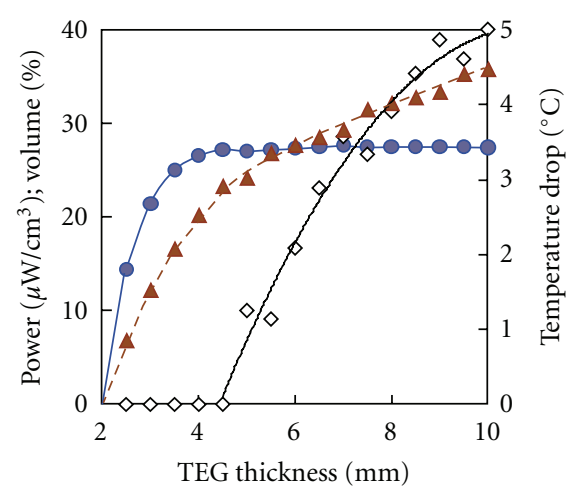

(c)

FIGURE 7: (a) Design of the simulated TEG module integrated into a piece of clothing (1). (b) Simulated dependence of the maximum power of this module (circles), and of the similar device, where the radiator has fins or pins (not shown in (a)), for a thickness of 5 to $10 \mathrm{~mm}$ (triangles). (c) Simulated dependence of the power per unit volume of the TEG (circles), the optimal temperature drop on the thermopile (triangles), and the volume of the module occupied by radiator on module thickness (diamonds). At a thickness of 2 to $4.5 \mathrm{~mm}$, the device has only two plates, thicker devices have a radiator with pins or fins occupying certain volume.

The simulation was performed at ambient temperature of $22^{\circ} \mathrm{C}$. It was assumed that the TEG module can be integrated somewhere on the human trunk, and the average heat transfer coefficient for a vertical plate with a characteristic length of $30 \mathrm{~cm}$ was used. Of course, the heat transfer on the bottom and top of vertical plate is different. However, the intention was to design the TEG module suitable for integration in any location on the trunk. Therefore, average conditions on the trunk were simulated. Figure 7(b) shows the calculated dependence of power on module thickness. As expected, the power increases in a thicker device because of decreased parasitic heat flow between its plates through air and supports. However, the dependence of power per unit volume showed clear maximum at a thickness of 4 to $5.5 \mathrm{~mm}$. Further increase of thickness results in decreased power per unit volume due to high thermal resistance of ambient air. It is beneficial to decrease the latter by a radiator through filling the cold plate with fins or pins. In such case, a part of the module volume is occupied by radiator. Therefore, the distance between plates decreases as compared to the design shown in Figure 7(a). The modeling of the TEG with a radiator was performed at different thicknesses of the module. In addition to optimizations discussed above, the optimum size of the radiator was found for every module thickness, Figure $7(\mathrm{c})$. Some scattering of points in Figure 7(c) is explained by a $0.5 \mathrm{~mm}$ step chosen for fin height. The power in a TEG with an optimized radiator size is also shown in Figure 7(b). The power, at least at a thickness of 5 to $10 \mathrm{~mm}$, linearly depends on module thickness. The dotted line for 3 to $5 \mathrm{~mm}$ thickness shows that even halfmillimeter-tall fins decrease power in modules thinner than $5 \mathrm{~mm}$.

We arrive to the conclusion on the principal design of a wearable TEG. A thin device, thinner than 5-7 mm, must have two plates, Figure $7(\mathrm{a})$, while the module thicker than $5-7 \mathrm{~mm}$ must have a radiator for maximum power generation. For plotting the optimum characteristics of a TEG in Figure 7(c), the results for a TEG with two plates are used for thicknesses of up to $4.5 \mathrm{~mm}$. For a thicker TEG, the results are shown for the case of a TEG with a radiator. The optimal volume of radiator for reaching maximum power is shown in Figure 7(c). It reaches $40 \%$ of the device volume in a $1 \mathrm{~cm}$-thick module. The optimum temperature drop on the thermopile at maximum power is also shown in Figure 7(c).

Based on the results shown in Figure 7(c), another simulation has been performed for the device shown in Figure 7(a), where the thickness of the module has been fixed at 3,4, and $6 \mathrm{~mm}$, but ambient temperature, and the corresponding thermal resistance of human being were variable. For the calculation of heat transfer coefficient, it was assumed that $30 \mathrm{~cm}$ of human body were located below the module. The thermal matching of the TEG has been performed at ambient temperature of $20^{\circ} \mathrm{C}$. Then, the performance characteristics of the module have been modeled at ambient temperatures between $0^{\circ} \mathrm{C}$ and $25^{\circ} \mathrm{C}$. The results of simulation of a TEG on the human leg, accounting for observed variation of the thermal resistance of the latter on ambient temperature, are shown in Figure 8.

For validation of calculated characteristics, the measurement of power generation on people has been performed using the TEG-4 that has a thickness of $6.5 \mathrm{~mm}$. It was integrated in jeans, on the anterior side of the left leg, at about $25 \mathrm{~cm}$ above the knee. The experimental results are shown in Figure 8, too. The other experimental results shown in Figure 8 correspond to average power production over 14 similar modules integrated on the front side of a shirt [16], and coincide well with the modeling results presented in this paper. However, as has been discussed above, in some locations on the chest, the thermal resistance of the body minimizes. Indeed, the TEG-4 placed in one of such locations has shown a power by a factor of two better, as registered at $23^{\circ} \mathrm{C}$, see Figure 8 .

The temperature drop and heat flow shown in Figure 8 are optimal and, in this case, the thermopile has optimal thermal resistance, according to (12). If its thermal resistance is increased above the required value, the temperature drop 




(a)

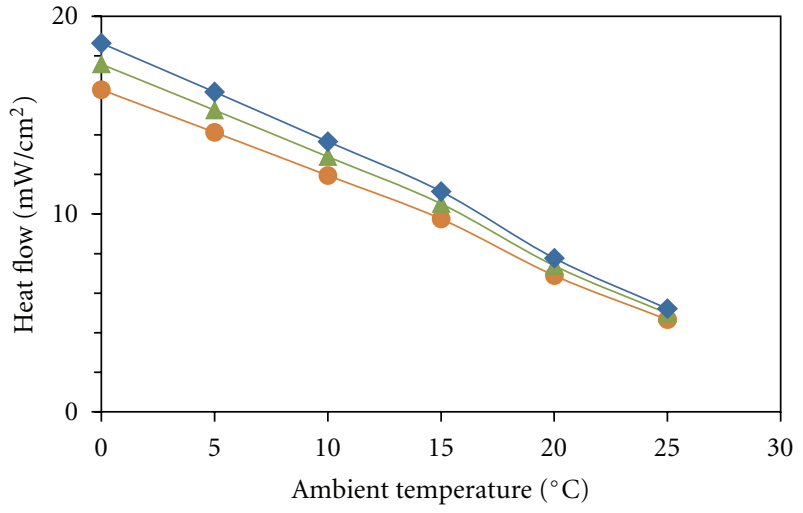

(b)



(c)

Figure 8: Simulated characteristics of the module of $3 \mathrm{~cm}$ in diameter at its different thickness, $3 \mathrm{~mm}$ (diamonds), $4 \mathrm{~mm}$ (triangles), and $6 \mathrm{~mm}$ (closed squares). The module is located on the leg of a subject and integrated in textile. Shown are calculated dependences of (a) the optimal temperature difference on the thermopile, (b) corresponding heat flow through the TEG, and (c) the maximum power per square centimeter of the module on temperature. Closed circles in (c) are measured with the TEG-4. Open circles show the average power measured in the TEG of electrocardiography system-in-a-shirt [16] that contains modules similar to the TEG-4. The open square shows maximum power reached by the TEG- 4 at $23^{\circ} \mathrm{C}$ in the best location found on the chest.

will increase, the heat flow will decrease, and vice versa. However, in such nonoptimal cases, the power will always decrease below the values shown in Figure 8(c). In the first approximation, any off-the-shelf thermopile with a $Z$ of $0.003 \mathrm{~K}^{-1}$, and with optimal thermal resistance, would show the characteristics close to those calculated in Figures 7 and 8. In case of a very small, for example, a micromachined, thermopile, the on-chip parasitic thermal resistance and electrical contact resistance between small semiconducting legs of thermopiles and metal interconnects can decrease power below the calculated values. The adverse effect of scaling of a thermopile in a TEG of fixed size on produced power has been analyzed in [17]. However, there are technological and design solutions to avoid or minimize this adverse effect of parasitic electrical and thermal resistances on power. Among them, one can mention the vacuum on-chip encapsulation of thermopiles [18] and increasing of contact area between metal and semiconductor [5]. Low-cost modern technology for thermopiles could enable competitive cost of body-powered devices as compared with batteries and bring such devices to the market.

\section{Conclusions}

The theory of wearable thermoelectric energy scavenger says that the thermal properties of human being must be accounted for the thermal optimization of such device. From the theory, such scavenger must have a thermal resistance of the order of hundreds $\mathrm{cm}^{2} \mathrm{~K} / \mathrm{W}$. The experiments performed in this work on measurements of heat flows and the thermal resistance of human body under a wearable device show that the thermal properties of the body depend on the thermal resistance of the attached TEG. This mutual influence of the human body and wearable TEG must also be accounted for the device optimization.

The measurements performed with the TEG-2 at ambient temperature of $-2^{\circ} \mathrm{C}$ have shown that a power of $1.2 \pm 0.2 \mathrm{~mW} / \mathrm{cm}^{2}$ can be produced unobtrusively, with no sensation of cold induced by the device. Most of the time people spend indoors where the temperature is maintained at a comfortable level by either heating or air conditioning. Therefore, on average, wearable TEG will not produce more than $10-30 \mathrm{~mW} / \mathrm{cm}^{2}$ in any climate. In a thin TEG of 
a few millimeters in thickness, which can be integrated in garments, the heat flow at typical indoor conditions is less than $10 \mathrm{~mW} / \mathrm{cm}^{2}$. The experiment shows that, in this situation, the thermal resistance of human being essentially increases locally under the device. Nevertheless, the modeling shows that such wearable TEG could still be competitive with a battery. The results of experiments coincide with the results of simulations. The results explain why the wearable TEGs supplied with a radiator $[1,2,5,12,16]$ demonstrate good power generation in self-powered devices. It is commonly believed that the radiator of a TEG only decreases the thermal resistance of ambient air: the larger its area of contact with air, the lower the thermal resistance of air, and the higher the heat flow. However, as has been shown in this work, the heat flow, increased due to radiator, affects (decreases) the local thermal resistance of human body under the TEG. This positive feedback results in further increase of heat flow locally and helps to produce more power in a wearable energy scavenger per its volume and per unit area on the skin.

\section{Acknowledgment}

This work has been performed in the frame of Human++ program of the Holst Centre and Imec on wearable wireless sensors and body area network.

\section{References}

[1] A. Bonfiglio and D. De Rossi, Eds., Wearable Monitoring Systems, Springer, New York, NY, USA, 2011.

[2] D. Rowe, Ed., CRC Handbook of Thermoelectrics, CRC Press, New York, NY, USA, 1994.

[3] V. Leonov and R. J. M. Vullers, "Wearable electronics selfpowered by using human body heat: the state of the art and the perspective," Journal of Renewable and Sustainable Energy, vol. 1, no. 6, Article ID 062701, 14 pages, 2009.

[4] E. A. Aydin and I. Guler, "Recent advances on bodyheat powered medical devices," Recent Patents on Biomedical Engineering, vol. 4, no. 1, pp. 33-37, 2011.

[5] V. Leonov, Y. van Andel, Z. Wang, R. J. M. Vullers, and C. Van Hoof, "Micromachined polycrystalline Si thermopiles in a Tshirt," Sensors \& Transducers Journal, vol. 127, no. 4, pp. 15-26, 2011.

[6] J. A. Harris and F. G. Benedict, "A biometric study of human basal metabolism," Proceedings of the National Academy of Sciences of the United States of America, vol. 4, no. 12, pp. 370373, 1918 .

[7] M. D. Mifflin, S. T. St. Jeor, L. A. Hill, B. J. Scott, S. A. Daugherty, and Y. O. Koh, "A new predictive equation for resting energy expenditure in healthy individuals," American Journal of Clinical Nutrition, vol. 51, no. 2, pp. 241-247, 1990.

[8] J. D. Hardy, A. P. Gagge, and J. A. J. Stolwijk, Eds., Physiological and Behavioral Temperature Regulation, Charles C. Thomas, Springfield, Ill, USA, 1970.

[9] S. Itoh, K. Ogata, and H. Yoshimura, Advances in Climatic Physiology, Springer, Berlin, Germany, 1972.

[10] J. Monteith and L. Mount, Eds., Heat Loss from Animals and Man, Butterworths, London, UK, 1974.
[11] V. Leonov, P. Fiorini, and R. J. M. Vullers, "Theory and simulation of a thermally matched micromachined thermopile in a wearable energy harvester," Microelectronics Journal, vol. 42, no. 4, pp. 579-584, 2011.

[12] T. Torfs, V. Leonov, and R. J. M. Vullers, "Pulse oximeter fully powered by human body heat," Sensors \& Transducers Journal, vol. 80, no. 6, pp. 1230-1238, 2007.

[13] V. Leonov, Z. Wang, R. Pellens, C. Gui, R. Vullers, and J. Su, "Simulations of a non-planar lithography and of performance characteristics of arcade microthermopiles for energy scavenging," in Proceeding of the 5th International Energy Conversion Engineering Conference (IECEC '07), St. Louis, Mo, USA, June 2007.

[14] M. Van Bavel, V. Leonov, R. F. Yazicioglu et al., "Wearable battery-free wireless 2-channel EEG systems powered by energy scavengers," Sensors \& Transducers Journal, vol. 94, no. 7, pp. 103-115, 2008.

[15] E. J. Carlson, K. Strunz, and B. P. Otis, "A $20 \mathrm{mV}$ input boost converter with efficient digital control for thermoelectric energy harvesting," IEEE Journal of Solid-State Circuits, vol. 45, no. 4, pp. 741-750, 2010.

[16] V. Leonov, T. Torfs, C. Van Hoof, and R. J. M. Vullers, "Smart wireless sensors integrated in clothing: an electrocardiography system in a shirt powered using human body heat," Sensors \& Transducers Journal, vol. 107, no. 8, pp. 165-176, 2009.

[17] V. Leonov, "Simulation of maximum power in the wearable thermoelectric generator with a small thermopile," Microsystem Technologies, vol. 17, no. 4, pp. 495-504, 2011.

[18] J. Xie, C. Lee, and H. Feng, "Design, fabrication, and characterization of CMOS MEMS-Based thermoelectric power generators," Journal of Microelectromechanical Systems, vol. 19, no. 2, pp. 317-324, 2010. 


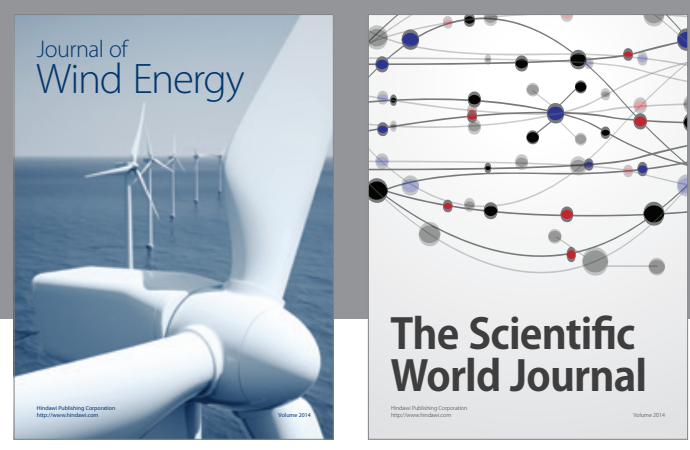

The Scientific World Journal

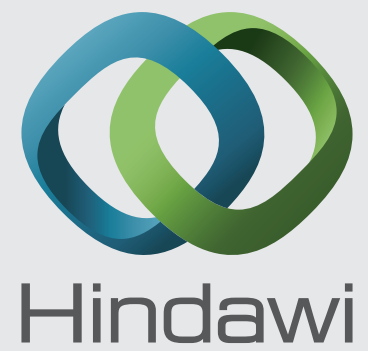

Submit your manuscripts at http://www.hindawi.com
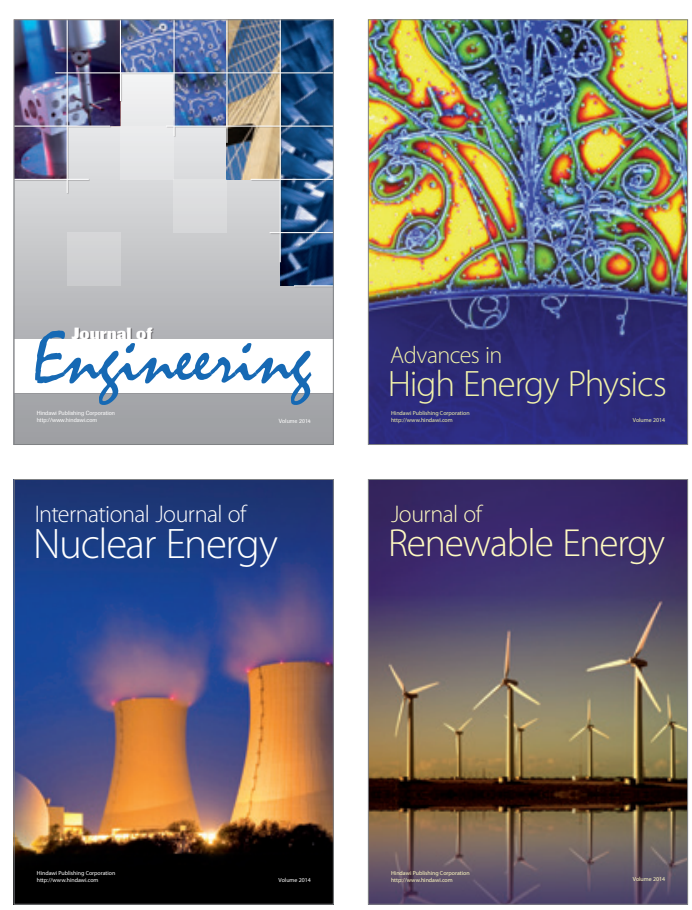

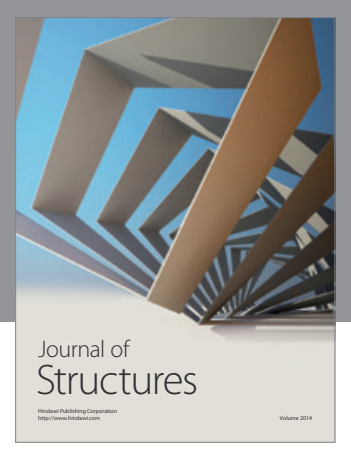

Rotating
Mechinery
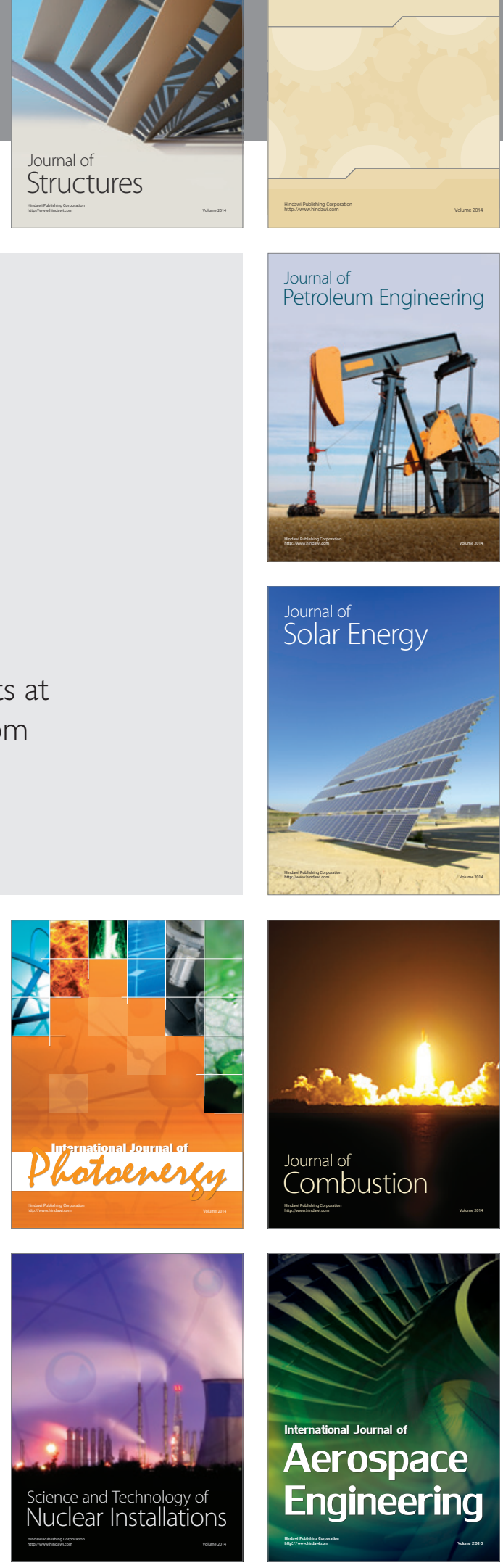